\title{
Multiscale Lung Texture Signature Learning Using the Riesz Transform
}

\author{
Adrien Depeursinge ${ }^{1,2}$, Antonio Foncubierta-Rodriguez ${ }^{1}$, \\ Dimitri Van de Ville ${ }^{2,3}$, and Henning Müller ${ }^{1,2}$ \\ 1 University of Applied Sciences Western Switzerland (HES-SO) \\ 2 University and University Hospitals of Geneva (HUG), Switzerland \\ 3 Ecole Polytechnique Fédérale de Lausanne (EPFL), Switzerland
}

http://medgift.hevs.ch/

\begin{abstract}
Texture-based computerized analysis of high-resolution computed tomography images from patients with interstitial lung diseases is introduced to assist radiologists in image interpretation. The cornerstone of our approach is to learn lung texture signatures using a linear combination of $N$-th order Riesz templates at multiple scales. The weights of the linear combination are derived from one-versus-all support vector machines. Steerability and multiscale properties of Riesz wavelets allow for scale and rotation covariance of the texture descriptors with infinitesimal precision. Orientations are normalized among texture instances by locally aligning the Riesz templates, which is carried out analytically. The proposed approach is compared with state-of-the-art texture attributes and shows significant improvement in classification performance with an average area under receiver operating characteristic curves of 0.94 for five lung tissue classes. The derived lung texture signatures illustrate optimal class-wise discriminative properties.
\end{abstract}

Keywords: Texture analysis, Riesz, steerability, interstitial lung diseases, high-resolution computed tomography, computer-aided diagnosis.

\section{Introduction}

Objective assessment of texture information is a difficult task in radiology [1]. Texture is central to human image understanding and plays an important role in efficient characterization of biomedical tissue that cannot be described in terms of shape or morphology [2]. Early detection of diffuse disease conditions requires to analyze very subtle changes in texture properties of the image, where computerized image processing proved to significantly outperform clinical experts [1]. The various appearances of lung tissue affected by interstitial lung diseases (ILD) in high-resolution computed tomography (HRCT) are best characterized in terms of texture properties [3]. Differentiation of these patterns is regarded as difficult even for experienced radiologists. Consequently, several studies investigated the potential of computerized classification of the lung parenchyma to assist radiologists in HRCT interpretation [4-7]. To ensure the success of such a system, the 
ability of the image attributes to encode the subtle texture signatures associated with the various lung tissue types is crucial. In particular, localized quantification of orientations and scales is known to be relevant for texture discrimination [8, 9]. Whereas most of the image analysis approaches to texture feature extraction are based on the characterization of these two affine properties, all require arbitrary sampling of at least one of these two parameters (e.g., grey-level co-occurrence matrices (GLCMs) 9], run-length matrices (RLE), local binary patterns (LBP) [8], and non-steerable Gabor or Gaussian filterbanks [10]). The choice of scales and orientations has a direct impact on system performance and is difficult since these properties vary for each image pixel.

In this article, we introduce a novel texture analysis approach allowing translation invariance as well as scale and rotation covariance with infinitesimal precision. It extends our previous work 11] by using support vector machines (SVM) to learn the respective relevance of multiscale Riesz components. Class-wise texture signatures are then obtained from linear combinations of the latter, allowing for visual assessment of the learned texture patterns.

\section{Material and Methods}

\section{$2.1 \quad$ Dataset}

A publicly available dataset of 85 ILD cases with annotated HRCT images is used to evaluate our approach [12]. Expert annotations were carried out in collaboration by two radiologists with 15 and 20 years of experience in CT imaging. The slice thickness is $1 \mathrm{~mm}$ and the inter-slice distance is $10 \mathrm{~mm}$. The images were acquired with two imaging devices at the Radiology Service of the University Hospitals of Geneva: a Philips Mx8000 IDT 16 CT Scanner and a General Electric HiSpeed CT. The five lung tissue classes encountered in most ILDs were chosen as lung texture classes: healthy $(\mathrm{H})$, emphysema $(\mathrm{E})$, ground glass $(\mathrm{G})$, fibrosis $(\mathrm{F})$ and micronodules $(\mathrm{M})$. In each annotated slice, 2D hand-drawn regions of interests (ROIs) are divided into $32 \times 32$ square blocks. The visual appearance of the lung texture classes and their distribution are detailed in Fig. 4 .

\subsection{Multiscale Lung Texture Signature Learning}

The cornerstone of our approach to multiscale lung texture signature learning is to use the structural risk minimization principle to derive class-wise texture prototypes from the Riesz transform. The obtained class-wise texture signature has optimal discriminative properties for a given one-versus-all (OVA) classification task. The Riesz transform yields steerable filterbanks and commutes with translation, scaling or rotation [13]. The richness of the filterbank is controlled by the order $N$ of the Riesz $\mathcal{R}$ transform as:

$$
\widehat{\mathcal{R}^{\left(n_{1}, n_{2}\right)} f}(\boldsymbol{\omega})=\sqrt{\frac{n_{1}+n_{2}}{n_{1} ! n_{2} !}} \frac{\left(-j \omega_{1}\right)^{n_{1}}\left(-j \omega_{2}\right)^{n_{2}}}{\|\boldsymbol{\omega}\|^{n_{1}+n_{2}}} \hat{f}(\boldsymbol{\omega}),
$$


$N=1$
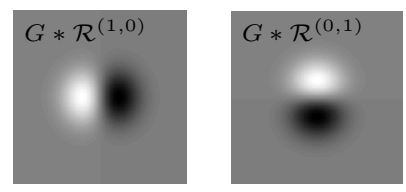

$N=3$
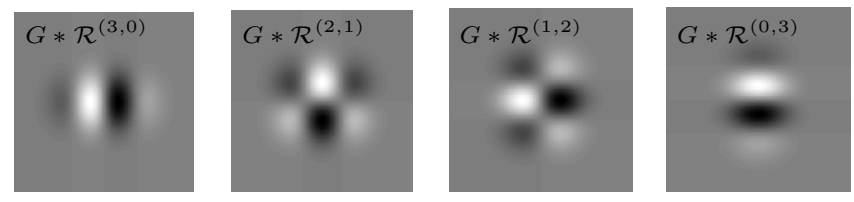

Fig. 1. Riesz filterbanks for $N=1,2,3$

for all combinations of $\left(n_{1}, n_{2}\right)$ with $n_{1}+n_{2}=N$ and $n_{1,2} \in \mathbb{N}$. The vector $\boldsymbol{\omega}$ is composed of $\omega_{1,2}$ corresponding to the frequencies in the two image axes, and $\hat{f}(\boldsymbol{\omega})$ denotes the Fourier transform of $f(\boldsymbol{x})$. The Riesz transform yields $N+1$ distinct components behaving as $N$-th order directional differential operators. Riesz components $\mathcal{R}^{\left(n_{1}, n_{2}\right)}$ convolved with isotropic Gaussian kernels $G(\boldsymbol{x})$ for $N=1,2,3$ are depicted in the spatial domain in Fig. 1. Multiscale versions of the filterbanks are obtained by coupling the Riesz transform with Simoncelli's multi-resolution framework based on isotropic band-limited wavelets [14]. Four scales $s_{i}=\{1, \ldots, 4\}$ with a dyadic progression are used to cover the Nyquist domain. The Riesz wavelet filterbanks are steerable, which means that the response of each component $G * \mathcal{R}^{\left(n_{1}, n_{2}\right)}$ rotated by an arbitrary angle $\theta$ can be derived analytically from a linear combination of the responses of all components of the filterbank [15, 13]. This property enables rotation covariance of the proposed texture descriptors with infinitesimal angular precision. To ensure that the distribution of the directional information is normalized among the Riesz components for any rotation of the texture patterns, each components are all locally aligned to maximize the response of $G * \mathcal{R}^{(N, 0)}$ at the finest scale, which is carried out analytically and proved to improve lung texture classification performance in [11]. This enables rotation invariance of the texture descriptors without discarding precious orientation information, which is often lost when using isotropic detectors [7, 6] or when averaging the responses of multi-oriented features as it is commonly carried out for GLCMs, RLEs and Gaussian filterbanks [5]. To summarize, the Riesz wavelets benefit from the steerability property while enabling much richer feature extraction than rotated filterbanks and classical steerable filterbanks [16]. Therefore, it allows multiscale and multi-directional image analysis with infinitesimal spatial and angular precision 1 .

In order to optimally exploit the richness of the feature detectors encompassed in the multiscale Riesz components for a given texture classification task, an

\footnotetext{
${ }^{1}$ In the discrete domain, the spatial and angular precisions are determined by Nyquist.
} 
appropriate weighting scheme of the energy of the responses of the multiscale Riesz components is required. The goal is to build an optimal texture signature $\Gamma_{c}^{N}$ of the class $c$ (versus all) from a linear combination of the multiscale Riesz components as:

$$
\Gamma_{c}^{N}=w_{1}\left(G * \mathcal{R}^{(N, 0)}\right)_{s_{1}}+w_{2}\left(G * \mathcal{R}^{(N-1,1)}\right)_{s_{1}}+\cdots+w_{4 N+4}\left(G * \mathcal{R}^{(0, N+1)}\right)_{s_{4}} .
$$

$l_{1}$-norm support vector machines (SVM) are used to find the optimal weights $\boldsymbol{w}^{\mathrm{T}}=\left(w_{1} \ldots w_{4 N+4}\right)$ in the sense of structural risk minimization [17] as:

$$
\min _{\boldsymbol{w}, \xi, b}\left\{\frac{\|\boldsymbol{w}\|_{1}^{2}}{2}+C \sum_{i=1}^{n} \xi_{i}\right\} \quad \text { subject to } \quad y_{i}\left(K\left(\boldsymbol{w}, \boldsymbol{x}_{\boldsymbol{i}}\right)-b\right) \geq 1-\xi_{i}, \quad \xi_{i} \geq 0 .
$$

where $\xi$ is the slack variable of the soft margin, $C$ is the cost of the errors, $\boldsymbol{x}_{\boldsymbol{i}}$ are the texture instances $i=1 \ldots n$ expressed in terms of the energy of the Riesz components, and $y_{i}$ are the corresponding labels. $K\left(\boldsymbol{x}_{\boldsymbol{i}}, \boldsymbol{x}_{\boldsymbol{j}}\right)$ is a Gaussian kernel as: $K\left(\boldsymbol{x}_{\boldsymbol{i}}, \boldsymbol{x}_{\boldsymbol{j}}\right)=\exp \left(\frac{-\left\|x_{i}-x_{j}\right\|_{1}^{2}}{2 \sigma^{2}}\right)$. The contribution of each Riesz component is determined by the weight that its corresponding energy level received in Eq. (3). For multiclass classification with $N_{c}$ classes, the OVA approach is used. The model with the highest decision level for the positive class determines the final class $c_{\max }$ as: $\max _{c \in\left\{1, \ldots, N_{c}\right\}}\left\{K\left(\boldsymbol{w}_{\boldsymbol{c}}, \boldsymbol{x}_{\boldsymbol{i}}\right)-b\right\}$. The global workflow of the proposed approach for lung texture signature extraction and classification is summarized in Fig. 2 .

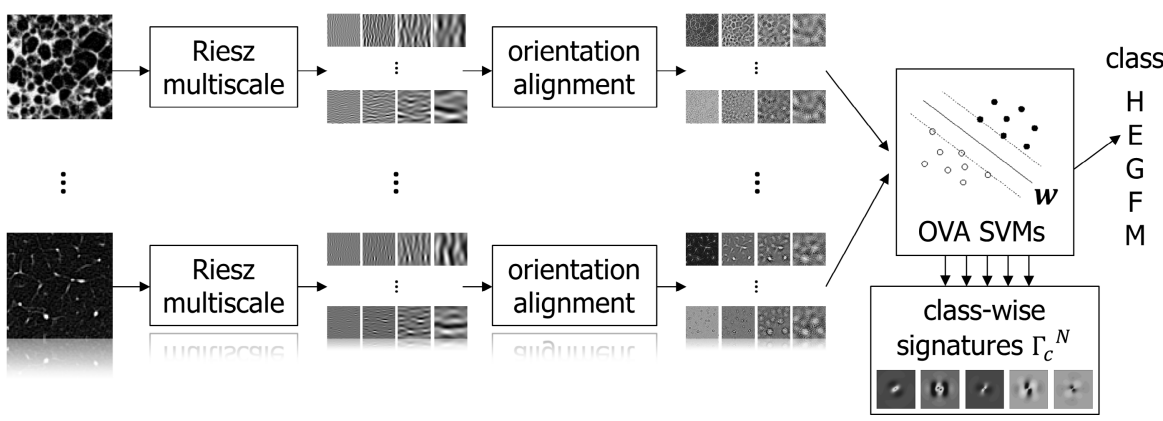

Fig. 2. Flow chart of lung texture signature learning and classification

\section{Results}

The proposed methods are evaluated both qualitatively and quantitatively on artificial and real lung textures. The principle of multiscale texture signature learning is first demonstrated on artificial data, where scale and rotation covariance are investigated in Fig. 3. All artificial texture patterns are containing noise and their signatures are learned when confronted to white noise. The first two 


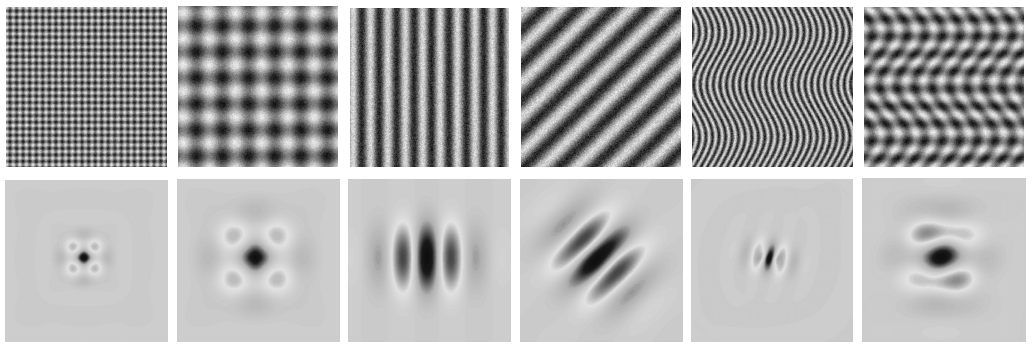

Fig. 3. Lower row: multiscale texture signatures $\Gamma_{c}^{8}$ of the upper row for $N=8$

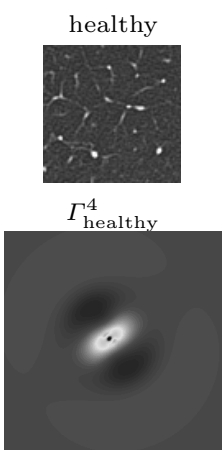

3011 blocks,

7 patients.

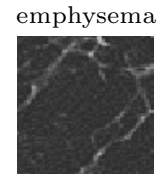

$$
\Gamma_{\mathrm{em}}^{4}
$$

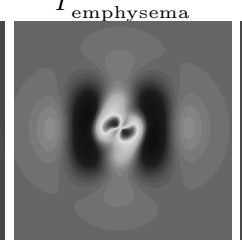

407 blocks,

6 patients.

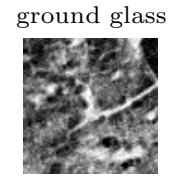

$$
\Gamma_{\mathrm{gr}}^{4}
$$

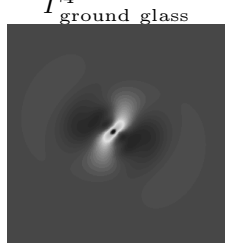

2226 blocks,

32 patients.

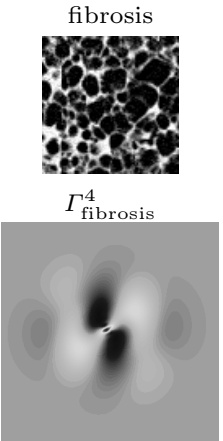

2962 blocks,

37 patients.
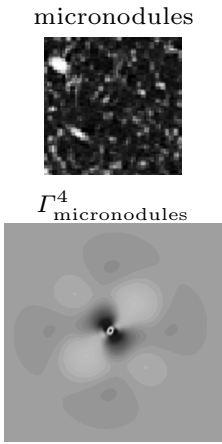

5988 blocks,

16 patients.

Fig. 4. Distribution of the texture classes and visual appearance of the class-wise multiscale lung texture signatures $\Gamma_{c}^{4}$

signatures are learned from two checkerboards with various scales. The distribution of the weights $\boldsymbol{w}$ for the scales $\left\{s_{1}, \ldots, s_{4}\right\}$ are $\{0.1 \%, 18.5 \%, 81.1 \%, 0.3 \%\}$ for the small scale and $\{2.3 \%, 3.9 \%, 14 \%, 79.8 \%\}$ for the large scale checkerboard. The rotation covariance is demonstrated with oriented stripes in the third and fourth columns of Fig. 3, Robustness to non-rigid transformations is illustrated with deformed versions of the stripes and checkerboard in the last two columns.

The visual appearance of the five lung tissue classes and the corresponding learned class-wise texture signatures over the entire dataset in OVA configurations are shown in Fig. 4 Fig. 5 shows the receiver operating characteristic (ROC) analysis of the classification performance of the proposed methods over the 85 folds of a leave-one-patient-out cross-validation. We compared our approach with two commonly used lung texture feature sets: LBPs [4] and GLCMs combined with RLEs [5]. We optimized the parameters of each approach using an exhaustive grid search. A radius $R \in\{1,2\}$ pixels and a number of samples $P \in\{8,16\}$ are used for LBPs, according to [4]. For GLCMs and RLEs, distances of $\{1,2, \ldots, 5\}$ are used and the texture measures from [9] are averaged across orientations of $\left\{0^{\circ}, 45^{\circ}, 90^{\circ}, 135^{\circ}\right\}$. A grey-level reduction of 8 levels obtained 

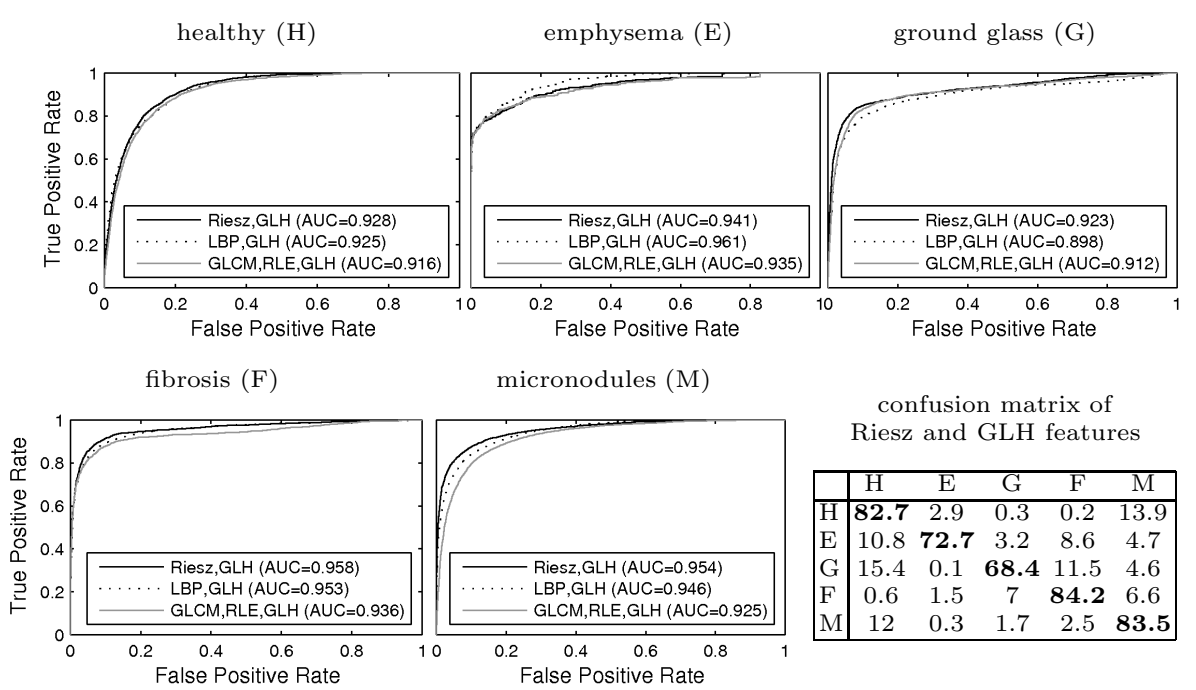

confusion matrix of Riesz and GLH features

\begin{tabular}{|c|ccccc|}
\hline & $\mathrm{H}$ & $\mathrm{E}$ & $\mathrm{G}$ & $\mathrm{F}$ & $\mathrm{M}$ \\
\hline $\mathrm{H}$ & $\mathbf{8 2 . 7}$ & 2.9 & 0.3 & 0.2 & 13.9 \\
$\mathrm{E}$ & 10.8 & $\mathbf{7 2 . 7}$ & 3.2 & 8.6 & 4.7 \\
$\mathrm{G}$ & 15.4 & 0.1 & $\mathbf{6 8 . 4}$ & 11.5 & 4.6 \\
$\mathrm{~F}$ & 0.6 & 1.5 & 7 & $\mathbf{8 4 . 2}$ & 6.6 \\
$\mathrm{M}$ & 12 & 0.3 & 1.7 & 2.5 & $\mathbf{8 3 . 5}$ \\
\hline
\end{tabular}

Fig. 5. ROC analysis for the various texture analysis approaches and confusion matrix. $N=4$ for all Riesz features.

best performance when compared to 16 and 32. All approaches are combined with 22 grey level histogram (GLH) bins in [-1050;600] Hounsfield Units (HU) and the percentage of air pixels with values $\leq-1000 \mathrm{HU}$. Best area under ROC curves (AUC) are of $0.941,0.936$ and 0.925 for Riesz $(N=4), \operatorname{LBPs}(R=1, P=8)$ and GLCMs with RLEs, respectively.

\section{Discussions and Conclusions}

We propose a novel texture analysis method to learn multiscale texture signatures based on Riesz wavelets and SVMs, which is rotation and scale covariant. A pixel-wise alignment of the Riesz templates ensures the normalization of the distribution of the directional information over the Riesz components, which allows both inter-instance rotation invariance and intra-instance rotation covariance, similarly to rotation-invariant LBPs [8]. The important scales and orientations are learned based on the structural risk minimization principle and therefore do not need a priori assumptions, which is an advantage when compared to other state-of-the-art texture features such as GLCMs, RLEs and LBPs. Linear combinations of multiscale Riesz components allow discovering class-wise important discriminatory patterns and visual analysis of their relevance. The multiscale texture signatures shown in Fig. 3 demonstrate the ability of our approach to characterize texture patterns with multiple and varying scales and orientations. A relative robustness to non-rigid transformations is also observed. The lung texture signatures depicted in Fig. 4 are showing important class-wise discriminative properties. $\Gamma_{\text {fibrosis }}^{4}$ clearly resembles the fibrosis patterns characterized by air bubbles surrounded by high-density walls of collagen. The same is true for 
$\Gamma_{\text {micronodules }}^{4}$, where the micronodule detector is clearly visible with a high peak in the center of the signature for relatively small scales. $\Gamma_{\text {healthy }}^{4}$ and $\Gamma_{\text {ground glass }}^{4}$ are found to be similar, which is coherent to the definition of ground glass characterized by a diffuse increased opacity, where the bronchovascular structures remain visible. $\Gamma_{\text {healthy }}^{4}$ and $\Gamma_{\text {ground glass }}^{4}$ are therefore implementing a hybrid ridge and peak detector corresponding to the projections of the bronchovascular structures in 2D HRCT slices. Emphysema patterns are the result of the destruction of lung tissue, which is replaced by air. This process does, therefore, not engender the typical texture signature that our method aims to learn. LBPs seem to better encode the transitions between air and parts of remaining tissue, which shows the potential of combining Riesz and LBP. ROC analysis of the classification performance of the texture analysis approaches reveals an excellent average performance $\mathrm{AUC}=0.94$ for the proposed approach, based on realistic data and methodology. It outperforms LBPs and GLCMs combined with RLEs in terms of overall classification performance with high statistical significance based on a 1tailed paired T-test: $p=4.75 \times 10^{-20}$ for Riesz versus LBPs and $p=5.59 \times 10^{-43}$ for Riesz versus GLCMs combined with RLEs. This performance suggests that it can provide valuable assistance in the difficult task of texture analysis of lung tissue patterns in clinical routine with high reliability. In future work, class-wise feature combination and selection among various Riesz orders and other texture features such as LBPs, GLCMs and RLEs will be investigated using SVM-based recursive feature elimination. We are also currently extending our approach to three dimensions. It is expected to provide even better results, since the number of possible scales and orientations increases exponentially in 3D. A priori knowledge on their organization in $3 \mathrm{D}$ is difficult to obtain, because textures existing in more than two dimensions cannot be fully visualized by humans.

Acknowledgments. This work was supported by the Swiss National Science Foundation (grants 205321-130046 and PP00P2-123438), the CIBM, and the EU in the context of Khresmoi (257528).

\section{References}

1. Wagner, R.F., Insana, M.F., Brown, D.G., Garra, B.S., Jennings, R.J.: Texture discrimination: radiologist, machine and man. In: Blakemore, C., Adler, K., Pointon, M. (eds.) Vision, pp. 310-318. Cambridge University Press (1991)

2. Tourassi, G.D.: Journey toward computer-aided diagnosis: Role of image texture analysis. Radiology 213(2), 317-320 (1999)

3. Webb, W.R., Müller, N.L., Naidich, D.P.: High-Resolution CT of the Lung. Lippincott Williams \& Wilkins, Philadelphia (2001)

4. Sørensen, L., Shaker, S.B., De Bruijne, M.: Quantitative analysis of pulmonary emphysema using local binary patterns. IEEE Transactions on Medical Imaging 29(2), 559-569 (2010)

5. Park, Y.S., Seo, J.B., Kim, N., Chae, E.J., Oh, Y.M., Lee, S.D., Lee, Y., Kang, S.H.: Texture-based quantification of pulmonary emphysema on high-resolution computed tomography: Comparison with density-based quantification and correlation with pulmonary function test. Investigative Radiology 43(6), 395-402 (2008) 
6. Sluimer, I.C., Prokop, M., Hartmann, I., van Ginneken, B.: Automated classification of hyperlucency, fibrosis, ground glass, solid, and focal lesions in highresolution CT of the lung. Medical Physics 33(7), 2610-2620 (2006)

7. Depeursinge, A., Van De Ville, D., Platon, A., Geissbuhler, A., Poletti, P.-A., Müller, H.: Near-affine-invariant texture learning for lung tissue analysis using isotropic wavelet frames. IEEE Transactions on Information Technology in BioMedicine (2012)

8. Ojala, T., Pietikäinen, M., Mäenpää, T.: Multiresolution gray-scale and rotation invariant texture classification with local binary patterns. IEEE Transactions on Pattern Analysis and Machine Intelligence 24(7), 971-987 (2002)

9. Haralick, R.M., Shanmugam, K., Dinstein, I.: Textural features for image classification. IEEE Transactions on Systems, Man and Cybernetics 3(6), 610-621 (1973)

10. Jain, A.K., Karu, K.: Learning texture discrimination masks. IEEE Transactions on Pattern Analysis and Machine Intelligence 18(2), 195-205 (1996)

11. Depeursinge, A., Foncubierta-Rodriguez, A., Van de Ville, D., Müller, H.: Lung Texture Classification Using Locally-Oriented Riesz Components. In: Fichtinger, G., Martel, A., Peters, T. (eds.) MICCAI 2011, Part III. LNCS, vol. 6893, pp. 231-238. Springer, Heidelberg (2011)

12. Depeursinge, A., Vargas, A., Platon, A., Geissbuhler, A., Poletti, P.-A., Müller, H.: Building a reference multimedia database for interstitial lung diseases. Computerized Medical Imaging and Graphics 36(3), 227-238 (2012)

13. Unser, M., Van De Ville, D.: Wavelet steerability and the higher-order Riesz transform. IEEE Transactions on Image Processing 19(3), 636-652 (2010)

14. Unser, M., Van De Ville, D., Chenouard, N.: Steerable pyramids and tight wavelet frames in $L_{2}\left(\mathbb{R}^{d}\right)$. IEEE Transactions on Image Processing 20(10), 2705-2721 (2011)

15. Freeman, W.T., Adelson, E.H.: The design and use of steerable filters. IEEE Transactions on Pattern Analysis and Machine Intelligence 13(9), 891-906 (1991)

16. Greenspan, H., Belongie, S., Goodman, R., Perona, P., Rakshit, S., Anderson, C.H.: Overcomplete steerable pyramid filters and rotation invariance. In: IEEE Computer Society Conference on Computer Vision and Pattern Recognition (CVPR), pp. 222-228 (1994)

17. Vapnik, V.N.: The Nature of Statistical Learning Theory. Springer, New York (1995) 